\title{
Comparison of endoscopic papillary large balloon dilation with or without endoscopic sphincterotomy for the treatment of large bile duct stones
}

\author{
Authors \\ Jin-Seok Park ${ }^{1,}{ }^{*}$, Seok Jeong ${ }^{1}$, Dong Ki Lee², Sung III Jang², Tae Hoon Lee ${ }^{3}$, Sang-Heum Park ${ }^{3}$, Jae Chul Hwang ${ }^{4}$, Jin \\ Hong Kim4, Byoung Moo Yoo ${ }^{4}$, Shin Goo Park ${ }^{5}$, Don Haeng Lee,
}

Institutions

1 Division of Gastroenterology, Department of Internal Medicine, Inha University School of Medicine, Incheon, South Korea

2 Yonsei University College of Medicine, Seoul, South Korea

3 Soon Chun Hyang University School of Medicine, Cheonan Hospital, Cheonan, South Korea

4 Ajou University School of Medicine, Suwon, South Korea

5 Occupational and Environmental Medicine, Inha University School of Medicine, Incheon, South Korea

submitted 8.1.2018

accepted after revision $\quad 17.5 .2018$

Bibliography

DOI https://doi.org/10.1055/a-0639-5147

Published online: 3.7.2018 | Endoscopy 2019; 51: 125-132

(c) Georg Thieme Verlag KG Stuttgart · New York

ISSN 0013-726X

\section{Corresponding author}

Seok Jeong, MD, Division of Gastroenterology, Department of Internal Medicine, Inha University Hospital, 27 Inhang-ro, Jung-gu, Incheon, 22332, Republic of Korea

Fax: +82-32-8902549

inos@inha.ac.kr

\section{ABSTRACT}

Background Endoscopic papillary large balloon dilation (EPLBD) without prior endoscopic sphincterotomy (EST) produces excellent outcomes for the treatment of large common bile duct (CBD) stones. However, it remains un- clear how the outcomes of EPLBD alone compare with those of EPLBD with EST. In this study, we assessed the safety and therapeutic outcomes of EPLBD with vs. without EST for the removal of large bile duct stones.

Methods This prospective, multicenter study was conducted on 200 patients with bile duct stones of $\geq 10 \mathrm{~mm}$ in diameter. Patients were randomly assigned to an EPLBD alone group $(n=100)$ or an EPLBD with EST group $(n=100)$. These two groups were compared with respect to overall procedure-related adverse events, overall stone removal success rate, number of endoscopic sessions required for complete stone removal, need for mechanical lithotripsy, and total procedure time.

Results The incidence of adverse events was not significantly different between the groups (EPLBD alone vs. EPLBD with EST: overall adverse events $6 \%$ vs. $4 \%, P=0.75$; pancreatitis $1 \%$ vs. $3 \%, P=0.62)$. Overall success $(P=0.35)$, initial success $(P=0.28)$, and the need for mechanical lithotripsy $(P=0.39)$ were also similar between groups. Median total procedure time tended to be greater in the EPLBD alone group (20.5 minutes) than in the EPLBD with EST group (18 minutes; $P=0.08$ ).

Conclusion The therapeutic outcomes and adverse events of EPLBD alone for the removal of large bile duct stones were comparable to those of EPLBD with EST.

Clinical Research Information Service at the Korean National Institute of Health

KCT0001564

TRIAL REGISTRATION: Multi-center, prospective, randomized controlled trial KCT0001564

at https://cris.nih.go.kr/cris

\section{Introduction}

Endoscopic sphincterotomy (EST) is a well-established treatment for common bile duct (CBD) stones and the procedure of choice for CBD stone removal [1]. However, the removal of large bile duct stones by conventional EST is difficult and large

\footnotetext{
* These authors contributed equally.
}

incisions are frequently required. Although large incisions are effective in terms of retrieving large biliary stones, they increase the risk of adverse events such as hemorrhage and perforation [2]. Ersoz et al. first reported the usefulness of large balloon dilation (12-20 mm in diameter) after EST for the removal of large bile duct stones and achieved stone clearance rates of up to $95 \%$ without the use of mechanical lithotripsy [1]. Many studies have since been conducted to verify the utili- 
ty of endoscopic papillary large balloon dilation (EPLBD), and have demonstrated the safety and feasibility of this technique [3-5]. Other studies have shown that hemorrhage and perforation might be reduced by EPLBD because dilation of the papilla is relatively slow [6], and that this technique may reduce the need for mechanical lithotripsy during the retrieval of large bile duct stones by providing a large biliary opening [7].

EST is generally recommended before EPLBD because prior EST is believed to prevent post-procedural pancreatitis by shifting the radial force generated by balloon dilation along the EST cutting direction toward the bile duct rather than the pancreatic duct, thus minimizing peripapillary edema after papilla dilation $[5,8]$. However, this hypothesis has not been sufficiently explored to determine the clinical usefulness and benefits of EST before balloon dilation. Furthermore, some authors have reported that EPLBD alone is effective for large bile duct stone removal and has acceptable adverse event rates $[9,10]$. However, the majority of previous investigations that compared EPLBD alone vs. EPLBD with EST were performed using small sample sizes [11,12], and thus, data available on the clinical aspects and the benefits of EST before EPLBD are limited. Accordingly, the current randomized study was undertaken to evaluate the efficacy and safety of EPLBD with EST vs. EPLBD alone for the removal of common bile duct stones.

\section{Methods}

This prospective, randomized, comparative study was conducted at four tertiary referral centers in South Korea by expert pancreaticobiliary endoscopists between November 2010 and October 2013. The study protocol was approved by the institutional review board of Inha University Hospital (IUH-IRB 0915), and all patients provided written informed consent before participating in the study. All authors had access to study data, and reviewed and approved the final manuscript. The study has been registered as a clinical trial (Cris.nih.go.kr number, KCT0001564).

\section{Patients}

Consecutive patients with a large $(\geq 10 \mathrm{~mm})$ CBD stone were enrolled. CBD stones were identified in all patients by imaging (abdominal ultrasonography, endoscopic ultrasonography, computed tomography, or magnetic resonance cholangiopancreatography). The study inclusion criteria were age $\geq 18$ years, maximum stone size $\geq 10 \mathrm{~mm}$, and distal CBD diameter $\geq 12 \mathrm{~mm}$. The exclusion criteria were: previous history of EST or endoscopic papillary balloon dilation; uncontrolled coagulopathy; acute pancreatitis; history of gastrointestinal surgery; concomitant pancreatic or biliary malignancy; benign biliary stricture; pregnancy or suspected pregnancy; the administration of specific medicines (protease inhibitor, protamine sulfate, somatostatin, morphine-like analgesics) from 2 days before to 2 days after the procedure; failure of selective biliary cannulation; and refusal to agree to the study protocol. Previously identified patient-related risk factors for post-endoscopic retrograde cholangiopancreatography (ERCP) pancreatitis (PEP), that is, young age (<60 years), female sex, and previous history of PEP, were also examined.

\section{Endoscopic procedures}

ERCP was performed using a side-viewing duodenoscope (TJF240, TJF-260V; Olympus Co., Tokyo, Japan). Before ERCP, patients were sedated with $5 \mathrm{mg}$ midazolam and $25 \mathrm{mg}$ meperidine hydrochloride (intravenously). Prophylactic antibiotics were routinely administered before ERCP, but protease inhibitors, which might affect the occurrence of PEP, were not administered either before or after ERCP. Selective CBD cannulation was achieved using a 0.035 -inch guidewire, and a diagnostic cholangiogram was obtained in all cases.

Randomization was performed after cholangiography confirmed the presence of a large stone in a dilated CBD (diameter $\geq 12 \mathrm{~mm}$ ). Patients were assigned randomly to an EPLBD alone group or an EPLBD with EST group using opaque sealed envelopes prepared by a third party. After achieving selective cannulation of the bile duct, a clinician opened the patient's envelope and performed the procedure indicated.

After sphincter dilation, stones were retrieved using a Dormia basket and/or an extraction balloon catheter ( $\triangleright$ Fig.1). When stone extraction was impossible using a conventional method, a mechanical lithotriptor (Lithotriptoren; MTW Endoskopie, Wesel, Germany) was used.

An occlusion cholangiogram was obtained at the end of the procedure to confirm complete CBD clearance. When a stone had not been completely removed, a nasobiliary drainage catheter or plastic stents were inserted to ensure biliary drainage, and repeat ERCP was performed every 2 or 3 days until complete stone removal had been achieved.

\section{Endoscopic sphincterotomy}

Limited sphincterotomy was performed before EPLBD using a 25-mm pull-type papillotome (CleverCut 3V; KD-V411 M, Olympus) and extended to a third of the total ampulla length. An electrosurgical unit (UES-30; Olympus) with a blended current was used at a power setting of $40 \mathrm{~W}$.

\section{Endoscopic papillary large balloon dilation}

EPLBD was performed using a 5-Fr hydrostatic balloon catheter (Microvasive; Boston Scientific Corp., Marlborough, Massachusetts, USA), with a maximum balloon diameter and length of $10-20 \mathrm{~mm}$ and $5.5 \mathrm{~cm}$, respectively, over a 0.035 -inch guidewire. After centering the balloon at the sphincter, the balloon was inflated slowly and incrementally up to $\geq 10 \mathrm{~mm}$ under low hydrostatic pressure using an inflation device (Indeflator; Abbott, Santa Clara, California, USA) and diluted contrast under fluoroscopic examination. EPLBD was terminated if "the waist sign" persisted in the inflated balloon when balloon inflation pressure reached $75 \%$ of the target pressure. After achieving the target diameter, the balloon inflation pressure was maintained for 60 seconds and then the balloon was deflated and removed. Balloon diameters were determined based on considerations of stone size, but did not exceed distal CBD diameter in order to avoid bile duct perforation. 

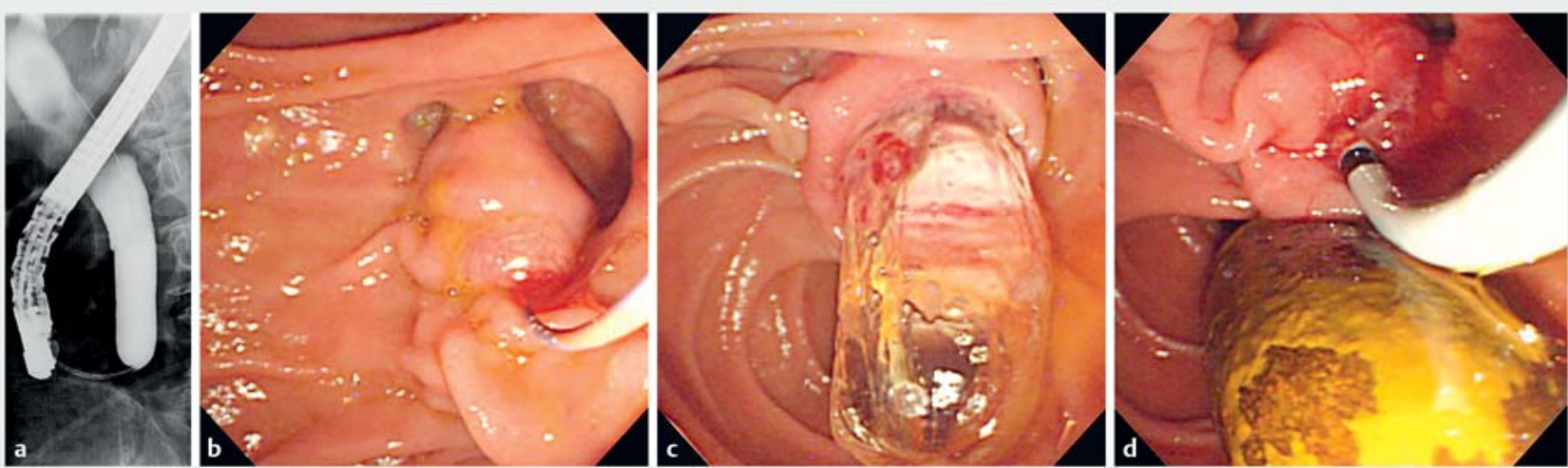

- Fig. 1 Endoscopic stone removal by endoscopic papillary large balloon dilation without sphincterotomy. a Fluoroscopic image. $\mathbf{b}$ - $\mathbf{d}$ Endoscopic images. b A guidewire positioned across the papilla. c A large balloon inflated across the papilla. $\mathbf{d}$ A large brown-pigmented stone extracted using a retrieval balloon catheter through the dilated papilla.

\section{Outcome measurements}

The primary outcome measure was overall adverse event rate, such as bleeding, perforation, asymptomatic hyperamylasemia, and pancreatitis. Secondary outcome measures were technical success rate, initial success rate, overall success rate, procedure time, number of ERCPs required for complete stone removal, and the need for mechanical lithotripsy.

Technical success was defined as the successful performance of EPLBD alone or EPLBD with EST. Complete stone clearance was defined as the absence of any filling defect, as determined by occlusion cholangiography conducted by an endoscopist after procedures. Initial success rate was defined as the rate of complete CBD clearance at the first ERCP session. Overall success rate was defined as the rate of complete CBD clearance irrespective of the number of ERCP sessions. Procedure time was defined as time from procedure start to endoscope removal.

Bleeding was defined as clinical evidence of bleeding, such as melena or hematemesis, with an associated hemoglobin concentration fall of $2 \mathrm{~g} / \mathrm{dL}$. PEP was defined as upper abdominal pain persisting for at least 24 hours and a serum amylase level the morning after surgery exceeding three times the upper limit of normal, as determined by 1991 consensus guidelines.

\section{Sample size calculation}

The study was conducted to evaluate the hypothesis that the safety of EPLBD alone is not inferior to that of EPLBD with EST. The study sample size was calculated as follows: level of significance $\alpha=0.05$; power $1-\beta=0.9$; noninferiority margin, $\delta=11 \%$ (based on an overall adverse event rate of $15 \%$ in a previous representative study on EPLBD with EST [1]); success rate $P=$ $90 \%$; and a dropout rate of $5 \%$. Using these data, the required sample size was calculated to be 97 patients per group.

\section{Statistical analysis}

Continuous variables are presented as medians and ranges. Categorical variables were compared using the chi-squared test and Fisher's exact test. Quantitative variables were compared using the Mann-Whitney test, and $P$ values of $<0.05$ were considered to indicate significance. The analysis was conducted using SPSS version 19.0 (IBM Corp., Armonk, New York, USA).

\section{Results}

\section{Patient demographic details}

During the study period, 209 patients were initially recruited. Five patients with a previous history of EST and one patient with suspected pancreatic cancer were recruited by mistake, and in three patients attempts to achieve selective biliary cannulation failed. Thus, nine patients were excluded ( $\mathbf{F i g . 2}$ ), and 200 patients with a CBD stone of $\geq 10 \mathrm{~mm}$ in maximum diameter were enrolled, and randomly and equally allocated to the EPLBD alone group or the EPLBD with EST group.

Baseline clinical characteristics are summarized in $>$ Table 1. Background demographic details were similar in the two groups. The median (range) stone size was $15.2 \mathrm{~mm}$ (14.1$16.2 \mathrm{~mm})$ in the EPLBD alone group and $14.6 \mathrm{~mm}(13.8-$ $15.5 \mathrm{~mm})$ in the EPLBD with EST group $(P=0.38)$. The median number of stones was $2(1-19)$ and $2(1-28 ; P=0.88)$, and the median CBD diameter was $17.8 \mathrm{~mm}(11.0-29.7 \mathrm{~mm})$ and $18 \mathrm{~mm}(10.0-45.0 \mathrm{~mm})$, respectively $(P=0.38)$.

\section{Adverse events}

Adverse events related to endoscopic procedures are summarized in $>$ Table 2. Overall adverse event rates were similar in the EPLBD alone and EPLBD with EST groups (6\% and $4 \%$, respectively; $P=0.75$ ). Three patients in the EPLBD alone group and one patient in the EPLBD with EST group experienced post-procedural abdominal pain $(P=0.62)$. Pancreatitis occurred in one patient $(1 \%)$ in the EPLBD alone group and in three patients (3\%) in the EPLBD with EST group $(P=0.62)$. In all cases, pancreatitis was of mild grade and all patients recovered unevent- 
Patients with large CBD stone $\mathbf{n}=209$

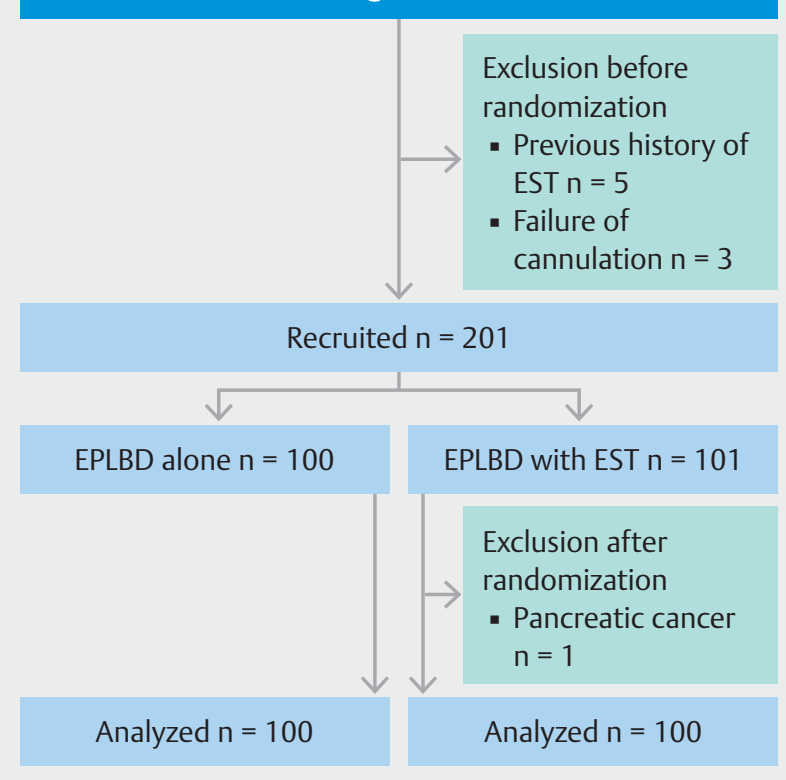

- Fig. 2 Flow diagram of the study design, showing the number of patients at each step. CBD, common bile duct; EPLBD, endoscopic papillary large balloon dilation; EST, endoscopic sphincterotomy.

fully with conservative treatment. No patient developed bleeding or perforation. In the EPLBD alone group, two patients experienced asymptomatic hyperamylasemia compared with none in the EPLBD with EST group.

\section{Clinical outcomes and endoscopic procedure}

The technical success rate was $100 \%$ in both groups. The median (range) balloon diameter used was $13.5 \mathrm{~mm}(11-19 \mathrm{~mm})$ for EPLBD alone and $13.5 \mathrm{~mm}(10-20 \mathrm{~mm})$ for EPLBD with EST ( $P=$ $0.24)$. CBD stones were extracted mainly using an extraction balloon catheter in both groups (EPLBD alone $76.1 \%$ and EPLBD with EST $72.7 \%$ ). A stone retrieval basket was required for 22 patients in the EPLBD alone group and for 24 patients in the EPLBD with EST group $(P=0.19)$. Complete stone removal rates were similar in the two groups ( $\vee$ Table 3 ). Stones were cleared during the first ERCP session in 77 patients in the EPLBD alone group and in 78 patients in the EBLBD with EST group $(P=0.28)$. Overall success rates in the EPLBD alone and EPLBD with EST groups were $92 \%$ and $88 \%$, respectively $(P=0.35)$. Failure to achieve complete CBD clearance occurred in 20 patients ( 8 in the EPLBD alone group and 12 in the EPLBD with EST group); causes of failure were stone impaction and incomplete stone capture using the basket despite mechanical lithotripsy. Stone removal in these 20 patients was achieved by percutaneous transhepatic cholangioscopy. Median total procedure time tended to be greater in the EPLBD alone group (20.5 minutes) than in the EPLBD with EST group (18 minutes; $P=0.08$ ).

\section{Discussion}

The results of this study indicate that EPLBD alone is equivalent to EPLBD with EST in terms of safety and efficacy for the treatment of patients with large CBD stones ( $\geq 10 \mathrm{~mm}$ ). EPLBD alone and EPLBD with EST both resulted in similarly high stone clearance rates, and overall adverse event rates in both groups were low and comparable. Stone-to-balloon ratio was found to inde-

- Table 1 Baseline clinical characteristics of the patients.

\begin{tabular}{|c|c|c|c|}
\hline Variable & $\begin{array}{l}\text { EPLBD alone } \\
(n=100)\end{array}$ & $\begin{array}{l}\text { EPLBD with EST } \\
(n=100)\end{array}$ & $P$ value \\
\hline Age, median (range), years & $74(56-91)$ & $73(49-91)$ & 0.42 \\
\hline Age $<60$ years, $\mathrm{n}(\%)$ & $1(1)$ & $6(6)$ & 0.05 \\
\hline Sex, male/female, $\mathrm{n}$ & $55 / 45$ & $48 / 52$ & 0.32 \\
\hline Previous history of PEP, $\mathrm{n}$ & 0 & 0 & \\
\hline Maximum transverse diameter of the largest CBD stone, median (range), mm & $15.2(14.1-16.2)$ & $14.6(13.8-15.5)$ & 0.38 \\
\hline No. of CBD stones, median (range) & $2(1-19)$ & $2(1-28)$ & 0.88 \\
\hline Maximum CBD diameter, median (range), mm & $17.8(11-29.7)$ & $18(10-45)$ & 0.38 \\
\hline \multicolumn{4}{|l|}{ Hematologic results, median (range) } \\
\hline - White blood cell count, $/ \mu \mathrm{L}$ & $9535(3330-145540)$ & $9465(2310-49120)$ & 0.48 \\
\hline - Total bilirubin, mg/dL & $2.25(0.2-12.3)$ & $2.4(2-20.4)$ & 0.70 \\
\hline - Aspartate aminotransferase, IU/L & $115(15-1770)$ & $72(10-1807)$ & 0.46 \\
\hline - Alanine aminotransferase, IU/L & $119.5(7-940)$ & $99.5(8-1194)$ & 0.80 \\
\hline - Alkaline phosphatase, IU/L & $422.5(11-3226)$ & $345.5(5-2523)$ & 0.11 \\
\hline
\end{tabular}


- Table2 Adverse events of endoscopic papillary large balloon dilation with or without endoscopic sphincterotomy.

\begin{tabular}{|l|l|l|}
\hline Variable & \multicolumn{1}{|c|}{$\begin{array}{l}\text { EPLBD alone } \\
(\mathbf{n = 1 0 0 )}\end{array}$} & $\begin{array}{l}\text { EPLBD with EST } \\
\mathbf{( n = 1 0 0 )}\end{array}$ \\
\hline Abdominal pain, $\mathrm{n}(\%)$ & $3(3)$ & $1(1)$ \\
\hline Pancreatitis, $\mathrm{n}(\%)$ & $1(1)$ & $3(3)$ \\
\hline Asymptomatic hyperamylasemia, $\mathrm{n}(\%)$ & $2(0)$ & 0.62 \\
\hline Perforation, $\mathrm{n}(\%)$ & $0(0)$ & $0(0)$ \\
\hline Bleeding, $\mathrm{n}(\%)$ & $0(0)$ & $0(0)$ \\
\hline Mortality, $\mathrm{n}(\%)$ & $0(0)$ & $0(0)$ \\
\hline EPLBD, endoscopic papillary large balloon dilation; EST, endoscopic sphincterotomy. & 0.50 \\
\hline
\end{tabular}

- Table 3 Comparison of clinical outcomes and endoscopic procedure between endoscopic papillary large balloon dilation with and without endoscopic sphincterotomy.

\begin{tabular}{|c|c|c|c|}
\hline Variable & $\begin{array}{l}\text { EPLBD alone } \\
(n=100)\end{array}$ & $\begin{array}{l}\text { EPLBD with EST } \\
(n=100)\end{array}$ & $P$ value \\
\hline Technical success rate, $\mathrm{n}(\%)$ & $100(100)$ & $100(100)$ & $>0.99$ \\
\hline Initial success for complete stone removal, n (\%) & $77(77)$ & $78(78)$ & 0.28 \\
\hline Overall success for complete stone removal, n (\%) & $92(92)$ & $88(88)$ & 0.35 \\
\hline Balloon diameter, median (range), mm & $13.5(11-19)$ & $13.5(10-20)$ & 0.24 \\
\hline Methods of stone extraction, $\mathrm{n}(\%)$ & $n=92$ & $\mathrm{n}=88$ & 0.19 \\
\hline - Extraction balloon & $70(76.1)$ & $64(72.7)$ & \\
\hline - Dormia basket & $4(4.3)$ & $7(8.0)$ & \\
\hline - Balloon with basket & $18(19.6)$ & $17(19.3)$ & \\
\hline Mechanical lithotripsy, n/N (\%) & $6 / 92(6.5)$ & $8 / 88(9.1)$ & 0.39 \\
\hline Number of endoscopic sessions, $n$ (\%) & $n=92$ & $\mathrm{n}=88$ & 0.25 \\
\hline . 1 & $77(83.7)$ & $78(88.6)$ & \\
\hline .2 & $14(15.2)$ & $7(8.0)$ & \\
\hline .3 & $1(1.1)$ & $3(3.4)$ & \\
\hline Total procedure time, median (range), minutes & $20.5(2.35-57.4)$ & $18.0(2.0-58.3)$ & 0.08 \\
\hline
\end{tabular}

pendently predict the requirement for mechanical lithotripsy, the use of which was significantly associated with the development of pancreatitis.

Procedural simplification and the maintenance of treatment efficacy are the developmental principles of new endoscopic treatment strategies. In this respect, EPLBD alone is a more attractive option than EPLBD with EST, because it is easier to perform, especially in patients with an anatomy unsuitable for EST, such as those who have undergone Roux-en-Y or Billroth II gastrectomy [12]. Furthermore, EPLBD alone is more suitable for patients with concomitant large stones and a bleeding tendency.

In the current study, EPLBD alone produced satisfactory results with respect to stone removal efficacy. The overall success rate of complete stone retrieval was $92 \%$, which compares fa- vorably with previously reported success rates of EPLBD with EST $(74 \%-99 \%)[3,5,8]$, and was not significantly different from that observed for EPLBD with EST in the current study ( $88 \% ; P=0.35)$. Nearly $80 \%$ of patients in both groups achieved complete ductal clearance in one endoscopic session, which is similar to that previously reported for EPLBD with EST [6]. In addition, no significant difference was observed in the need for mechanical lithotripsy (EPLBD alone $6.5 \%$ and EPLBD with EST $9.1 \%)$. These findings suggest that EPLBD alone provides sufficient orifice dilation for stone removal, and that it is a reasonable alternative treatment for the removal of large CBD stones. These results concur with those of a previous retrospective study, in which 131 patents with large CBD stones were treated by EPLBD alone $(n=62)$ or by EPLBD with EST $(n=69)$, and over- 
all successful stone removal rates were not significantly different (96.8\% and $95.7 \%$, respectively) [11].

Theoretically, EPLBD alone is easier to perform than EPLBD with EST, and thus, would be expected to shorten procedure times. In a retrospective comparative study, procedure times from successful CBD cannulation to complete stone removal were found to be significantly shorter for EPLBD alone than for EPLBD with EST (21.5 vs. 17.3 minutes; $P=0.04$ ) [13]. However, in the current study, total procedure time was longer for EPLBD alone (20.5 minutes) than for EPLBD with EST (18.0 minutes; $P=$ 0.08 ), which probably reflects the number of endoscopic sessions required for complete stone removal; $16.3 \%$ of patients in the EPLBD group and $11.4 \%$ of patients in the EPLBD with EST group required multiple endoscopic sessions. These findings demonstrate that EPLBD alone does not reduce total procedure time compared with EPLBD with EST.

Debate regarding the safety of EPLBD for the treatment of large CBD stones has continued since the technique was introduced in 2003, especially with respect to PEP [14]. Although the mechanisms of pancreatitis after EPLBD are unclear, the risk of EPLBD-related pancreatitis is believed to be related to pressure loading of the orifice of the main pancreatic duct during balloon dilation and consequent pancreatic duct obstruction [15-17]. Thus, performing EST prior to EPLBD might reduce the incidence of pancreatitis because it separates pancreatic and biliary orifices and directs balloon dilation forces away from the pancreatic duct $[1,18]$. However, the current study showed that PEP is rare after EPLBD regardless of EST, and that the two techniques have similar PEP rates (EPLBD alone $n=1$; EPLBD with EST $\mathrm{n}=3 ; P=0.62$ ). These results are supported by the findings of a previous comparative, retrospective study on EPLBD alone vs. EPLBD with EST conducted by Hwang et al., in which the PEP rates of EPLBD with EST and EPLBD alone were found to be similar $(P=0.59)$ [11]. Therefore, we suggest that performing EST before EPLBD will probably not reduce the rate of pancreatitis substantially.

Most researchers in Eastern populations have reported no direct link between endoscopic balloon dilation and pancreatitis risk, and our results concur. However, studies in Western populations have reported significantly higher incidences of PEP after endoscopic balloon dilation [19]. In our opinion, this phenomenon is probably due to racial differences rather than to variabilities in the techniques used for balloon dilation of the biliary orifice or stone extraction techniques. Sphincter of Oddi dysfunction (SOD) is a benign noncalculous obstructive disorder encountered at the level of the Sphincter of Oddi that causes pancreaticobiliary-type pain. Clinical suspicion of SOD before ERCP has been reported to be a strong independent predictor of PEP (a PEP rate of $19.1 \%$ was reported when SOD was suspected) [20]. The pathogenesis of SOD in a background of developing pancreatitis is not well understood, but it may be caused by poor outflow of pancreatic juice caused by post-procedural spasm of the Sphincter of Oddi. No study has been conducted on the prevalence of SOD in normal populations, and thus, it is not clear whether the prevalence of SOD is race dependent. However, it is generally accepted that the prevalence of SOD in Western countries is higher than in Asia. In a large sin- gle-center series (Indianapolis, USA) involving 1241 patients with recurrent pancreatitis, SOD (40.3\%) was found to be the most common etiology of recurrent pancreatitis [21]. Conversely, according to reports issued in Asian countries [22, 23], biliary microlithiasis is the most common cause of recurrent pancreatitis and SOD is unusual. Therefore, different prevalences of SOD in the West and East might underlie different PEP incidences after balloon dilation.

Differences between the nature of the stones extracted in Asian and Western populations might also affect the incidence of PEP. In the West, the majority of CBD stones are cholesterol stones that originate from the gallbladder and fewer than $10 \%$ of CBD stones are formed de novo within the CBD. In contrast, in Asia, because of a higher incidence of chronic biliary tree infection and infestation, pigment stones are much more common [24]. Unlike cholesterol stones, pigment stones are often soft and easily broken [25], and thus, clearance is often achieved by retrieval balloon sweeping and using a basket, even in cases of inadequate papilla dilation; whereas, for cholesterol stones, especially large stones, complete clearance frequently requires mechanical lithotripsy and multiple endoscopic sessions, which are potential risk factors for PEP.

In the current study, no clinically significant hemorrhage was encountered in either group. Minor intraprocedural bleeding was noted in some cases during EPLBD but this was controlled easily in all cases by spraying diluted epinephrine solution. Delayed bleeding did not occur in any patient. In previous studies, bleeding rates during EPLBD have been reported to vary between $0 \%$ and $9 \%[1,8,18,26]$, and bleeding rates have been reported to be significantly higher for EPLBD with large EST (8.3\%-9\%) than for EPLBD alone or EPLBD with minor EST [1, 4]. In the current study, the extent of ampullary incision during prior EST was limited to a third of the total ampulla length. We believe that both EPLBD with limited EST and EPLBD alone are safe with respect to procedure-related bleeding.

Perforation is a rare but the most serious adverse event after EPLBD. Although almost all patients who develop retroperitoneal perforation recover with medical treatment, perforation often requires surgical treatment and is potentially life threatening [27]. In the current study, perforation was not encountered in either group. To prevent perforation, we used a cautious balloon inflation technique, whereby the balloon was gradually inflated up to the target diameter, and if a balloon waist was observed in the distal CBD during inflation, no further pressure was applied until the waist disappeared. Generally, we find that the balloon waist disappears after waiting for about 1 minute, but if the waist does not disappear, we suggest conversion to an alternative stone retrieval method or repeat ERCP, because failure to disappear suggests the presence of an invisible biliary stricture.

Having achieved good results of EPLBD for large CBD stone removal, some investigators have tried to extend the indication of EPLBD to patients with large stones, a tapered distal bile duct, or stricture of the distal bile duct $[3,8]$. However, perforation, which is the most serious adverse event of EPLBD, is more likely to occur in patients with distal bile duct stricture. In a retrospective study of 945 patients from South Korea who under- 
went large CBD stone removal by EPLBD, it was reported that distal CBD stricture independently predicted perforation (odds ratio 17.08; $P<0.001$ ) [28]. Therefore, we suggest that the presence of a distal CBD stricture should be considered a relative contraindication to EPLBD. In addition, EPLBD alone would be more effective in patients with a predicted dilated CBD diameter greater than that of the stone (due to the risk of bile duct perforation). In cases with insufficient ampulla dilation, mechanical lithotripsy or multiple endoscopic sessions would be required to achieve complete CBD clearance. In the current study, mechanical lithotripsy was required to remove stones in 14 patients, and stone diameter to CBD diameter ratios tended to be larger in patients who required mechanical lithotripsy (median ratio 1.2 , range $0.88-2.5$ ) than in those who did not (median 1.0, range $0.4-2.0$ ), although this result was not statistically significant $(P=0.28)$. Therefore, we recommend that EPLBD alone is used in patients with a maximum CBD diameter large enough to accommodate the largest stone.

The current study has several limitations that require consideration. First, intent-to-treat analysis was not conducted, and thus, our results are subject to the effects of crossover and dropout. However, the aim of this study was to compare the adverse event rates and effectiveness of EPLBD with EST vs. EPLBD alone. Thus, the intent-to-treat approach was not actually required because the study was designed to provide information about the potential effects of treatment policy rather than on information on the potential effects of specific treatments. Second, the presence of a tapered CBD can also cause stone removal difficulties despite adequate balloon dilation [5], and we did not address this topic. Third, a large CBD stone was defined as a stone diameter of $\geq 10 \mathrm{~mm}$ because many authors have defined CBD stones $>10-15 \mathrm{~mm}$ in diameter as "large." However, no consensus has been reached on the definition of a "large CBD stone." Based on clinical experience, a stone of $10-$ $11 \mathrm{~mm}$ can be removed effectively using conventional methods even if EPLBD is not used. In the current study, the median largest CBD stone diameter was $13.6 \mathrm{~mm}$, but 40 patients ( $20 \%$ ) had a largest stone that was $<11 \mathrm{~mm}$. Therefore, it is possible that the current study exaggerates the efficacy of EPLBD regardless of EST. Finally, long-term outcomes of EPLBD with EST and EPLBD alone were not evaluated.

In conclusion, the current study shows that procedural success and adverse event rates of EPLBD without EST are comparable to those of EPLBD with EST, and thus, indicate that EPLBD alone could be considered a useful alternative modality for the treatment of large CBD stones.

\section{Acknowledgment}

This work was supported by an Inha University Hospital Research Grant.

\section{Competing interests}

None
References

[1] Ersoz G, Tekesin O, Ozutemiz AO et al. Biliary sphincterotomy plus dilation with a large balloon for bile duct stones that are difficult to extract. Gastrointest Endosc 2003; 57: 156 - 159

[2] Minami A, Nakatsu T, Uchida N et al. Papillary dilation vs sphincterotomy in endoscopic removal of bile duct stones a randomized trial with manometric function. Dig Dis Sci 1995; 40: 2550 - 2554

[3] Itoi T, Itokawa F, Sofuni A et al. Endoscopic sphincterotomy combined with large balloon dilation can reduce the procedure time and fluoroscopy time for removal of large bile duct stones. Am J Gastroenterol 2009; 104: $560-565$

[4] Maydeo A, Bhandari S. Balloon sphincteroplasty for removing difficult bile duct stones. Endoscopy 2007; 39: 958-961

[5] Misra S, Dwivedi M. Large-diameter balloon dilation after endoscopic sphincterotomy for removal of difficult bile duct stones. Endoscopy 2008; 40: 209-213

[6] Oh M], Kim TN. Prospective comparative study of endoscopic papillary large balloon dilation and endoscopic sphincterotomy for removal of large bile duct stones in patients above 45 years of age. Scand J Gastroenterol 2012; 47: 1071 - 1077

[7] Kim TH, Oh HJ, Lee JY et al. Can a small endoscopic sphincterotomy plus a large-balloon dilation reduce the use of mechanical lithotripsy in patients with large bile duct stones? Surg Endosc 2011; 25: 3330 3337

[8] Heo JH, Kang DH, Jung $\mathrm{HJ}$ et al. Endoscopic sphincterotomy plus large-balloon dilation versus endoscopic sphincterotomy for removal of bile-duct stones. Gastrointest Endosc 2007; 66: 720-726

[9] Chan H, Lai K, Lin C et al. Endoscopic papillary large balloon dilation alone without sphincterotomy for the treatment of large common bile duct stones. BMC Gastroenterology 2011; 11: 69

[10] Jeong S, Ki S, Lee DH et al. Endoscopic large-balloon sphincteroplasty without preceding sphincterotomy for the removal of large bile duct stones: a preliminary study. Gastrointest Endosc 2009; 70: 915-922

[11] Hwang JC, Kim JH, Lim SG et al. Endoscopic large-balloon dilation alone versus endoscopic sphincterotomy plus large-balloon dilation for the treatment of large bile duct stones. BMC Gastroenterol 2013; 13: 15

[12] Kogure H, Tsujino T, Isayama $\mathrm{H}$ et al. Short-and long-term outcomes of endoscopic papillary large balloon dilation with or without sphincterotomy for removal of large bile duct stones. Scand J Gastroenterol 2013; 49: $121-128$

[13] Li QL, Gao WD, Zhang C et al. Is endoscopic sphincterotomy plus large-balloon dilation a better option than endoscopic large-balloon dilation alone in removal of large bile duct stones? A retrospective comparison study Indian J Cancer 2015; 51: e13-17

[14] Baron TH, Harewood GC. Endoscopic balloon dilation of the biliary sphincter compared to endoscopic biliary sphincterotomy for removal of common bile duct stones during ERCP: a meta-analysis of randomized, controlled trials. Am J Gastroenterol 2004; 99: 1455-1460

[15] Mac Mathuna P, Siegenberg D, Gibbons D et al. The acute and longterm effect of balloon sphincteroplasty on papillary structure in pigs. Gastrointest Endosc 1996; 44: 650-655

[16] Ohashi A, Tamada K, Tomiyama T et al. Epinephrine irrigation for the prevention of pancreatic damage after endoscopic balloon sphincteroplasty. J Gastroenterol Hepatol 2001; 16: 568-571

[17] Akashi R, Kiyozumi T, Tanaka T et al. Mechanism of pancreatitis caused by ERCP. Gastrointest Endosc 2002; 55: 50- 54

[18] Attasaranya S, Cheon YK, Vittal $\mathrm{H}$ et al. Large-diameter biliary orifice balloon dilation to aid in endoscopic bile duct stone removal: a multicenter series. Gastrointest Endosc 2008; 67: 1046-1052 
[19] Weinberg B, Shindy W, Lo S. Endoscopic balloon sphincter dilation (sphincteroplasty) versus sphincterotomy for common bile duct stones. Cochrane Database Syst Rev 2006: CD004890

[20] Vandervoort J, Soetikno RM, Tham TC et al. Risk factors for complications after performance of ERCP. Gastrointest Endosc 2002; 56: 652 656

[21] Fischer M, Hassan A, Sipe BW et al. Endoscopic retrograde cholangiopancreatography and manometry findings in 1,241 idiopathic pancreatitis patients. Pancreatology 2010; 10: 444-452

[22] Kim HS, Moon JH, Choi HJ et al. The role of intraductal US in the management of idiopathic recurrent pancreatitis without a definite cause on ERCP. Gastrointest Endosc 2011; 73: $1148-1154$

[23] Takuma K, Kamisawa T, Hara S et al. Etiology of recurrent acute pancreatitis, with special emphasis on pancreaticobiliary malformation. Adv Med Sci 2012; 57: 244-250
[24] Kim DI, Kim M, Lee SK et al. Risk factors for recurrence of primary bile duct stones after endoscopic biliary sphincterotomy. Gastrointest Endosc 2001; 54: $42-48$

[25] Gregg JA, De Girolami P, Carr-Locke DL. Effects of sphincteroplasty and endoscopic sphincterotomy on the bacteriologic characteristics of the common bile duct. Am J Surg 1985; 149: 668-671

[26] Bang S, Kim MH, Park JY et al. Endoscopic papillary balloon dilation with large balloon after limited sphincterotomy for retrieval of choledocholithiasis. Yonsei Med J 2006; 47: 805-810

[27] Youn YH, Lim HC, Jahng JH et al. The increase in balloon size to over $15 \mathrm{~mm}$ does not affect the development of pancreatitis after endoscopic papillary large balloon dilatation for bile duct stone removal. Dig Dis Sci 2011; 56: $1572-1577$

[28] Park SJ, Kim JH, Hwang JC et al. Factors predictive of adverse events following endoscopic papillary large balloon dilation: results from a multicenter series. Dig Dis Sci 2013; 58: 1100-1109 